\title{
Féeries
}

Études sur le conte merveilleuX, XVII $-\mathrm{XIX}{ }^{\mathrm{e}}$ siècle

$11 \mid 2014$

L'illustration des contes

\section{Les fiancés-animaux illustrés du Cabinet des fées}

«Traduction » de Clément-Pierre Marillier pour deux contes de Madame d'Aulnoy : « Serpentin Vert » et « Le Prince Marcassin »

Betrothed Animals in the Cabinet des fées. Clément-Pierre Marillier's

Translation of Two Tales by Madame d'Aulnoy: "Serpentin Vert" and "Le Prince

Marcassin"

Swann Paradis

\section{CpenEdition}

Journals

Édition électronique

URL : http://journals.openedition.org/feeries/944

DOI : $10.4000 /$ feeries. 944

ISSN : 1957-7753

Éditeur

UGA Éditions/Université Grenoble Alpes

Édition imprimée

Date de publication : 19 décembre 2014

Pagination : 171-198

ISBN : 978-2-84310-281-3

ISSN : $1766-2842$

Référence électronique

Swann Paradis, "Les fiancés-animaux illustrés du Cabinet des fées », Féeries [En ligne], 11 | 2014, mis en ligne le 19 décembre 2015, consulté le 30 octobre 2020. URL : http://journals.openedition.org/ feeries/944; DOI : https://doi.org/10.4000/feeries.944

(C) Féeries 


\title{
Swann Paradis
}

Collège universitaire Glendon York University

\section{LES FIANCÉS-ANIMAUX ILLUSTRÉS DU CABINET DES FÉES}

\author{
«TRADUCTION» DE CLÉMENT-PIERRE MARILLIER POUR DEUX \\ CONTES DE MADAME D’AULNOY : «SERPENTIN VERT»
}

ET «LE PRINCE MARCASSIN"

$L$

ORSQUE LES FÉES DÉCIDENT DE CHÂTIER, elles le font souvent par la métamorphose animale, péripétie récurrente dans le schéma narratif qui sous-tend plusieurs contes. L'animalité devient alors «le centre même du récit, et le thème $\operatorname{principal~}^{\mathrm{I}}$ ", qui permet le plus souvent de "souligner la vulnérabilité, la déchéance physique, l'avilissement du héros ${ }^{2} »$. S’il existe une littérature immense sur cette question de la métamorphose - notamment en ce qui a trait aux thèmes du double, de l'androgyne ou du travestissement, ou encore au lien étroit qui la relie au concept de "transfert» en psychanalyse, et conséquemment à cette transformation de soi en l'autre, nécessaire à la constitution de toute identité - , les études que nous avons consultées ${ }^{3}$ portant tant sur les contes de fées en général, que sur ceux de Madame d'Aulnoy en particulier, insistent peu sur la représentation iconographique de ce passage d'une forme à une autre dans les ouvrages d'Ancien Régime. Or, si le privilège de l'image est de n'obliger à aucun sens de lecture, si sa polysémie rend illusoire l'épuisement du sens qui oscille constamment entre le figuratif et le symbolique, entre «images de savoir» et «images de plaisir» ${ }^{4}$, quelles pourraient

I. J. Bloch, «Le héros animal dans les contes de fées de Madame d'Aulnoy. Le Prince Marcassin, Serpentin Vert, La Chatte blanche, La Biche au bois", Dix-huitième siècle, $\mathrm{n}^{\circ}$ 42, 2010 , p. 120.

2. V. Meunier, "Le bestiaire fantastique», dans O. Piffault (dir.), Il était une fois... les contes de fées, 200I, p. 376.

3. Outre les ouvrages mentionnés dans cet article, pour un état des lieux exhaustif, on consultera avec profit: C. Ionescu (dir.), Book Illustration in the Long Eighteenth Century. Reconfiguring the Visual Periphery of the Text, 20II, p. I-50.

4. Nous reprenons la division suggérée par A.-M. Bassy, «Le texte et l'image», dans H.-J. Martin et R. Chartier (dir.), Histoire de l'édition française. Le livre triomphant I660-I830, t. II, I984, p. I48-I56. 
être alors les modalités de cette bipolarité s'exprimant dans la figuration de la métamorphose animale, telle qu'elle se présente sur les planches commandées à Clément-Pierre Marillier (I740-1808) pour l'anthologie du Cabinet des fées, éditée entre 1785 et 1789 par Charles-Joseph de Mayer? Dans le cadre du présent article, nous nous sommes proposé d'une part de prolonger la réflexion théorique sur "Le temps de la métamorphose» amorcée par Aurélia Gaillard's, et d'autre part de nous concentrer sur les "héros animaux ${ }^{6}$ " qui peuplent les contes de Madame d'Aulnoy illustrés par Marillier dans Le Cabinet des fées. D'autre part, cette sélection en quelque sorte imposée par l'occurrence de dessins dans l'anthologie appelle la terminologie associée au cycle des fiancés-animaux ${ }^{7}$ sur laquelle nous nous appuierons pour développer notre propre analyse iconographique, en nous concentrant sur les deux planches suivantes qui renvoient à la sous-catégorie du «fiancé-animal», représentative de "l'animalité négative», pour reprendre la catégorisation proposée par Anne Defrance ${ }^{8}$ : "Serpentin Vert» (Cat. II, CDF 03-2) et "Le Prince Marcassin» (Cat. I5, CDF 04-3) ${ }^{9}$. Nous tenterons de déterminer si les planches qui illustrent ces contes mettant en scène des héros-animaux issus d'une métamorphose procèdent d'une insertion ${ }^{\text {10 }}$ qui suggère autant qu'elle signifie; en d'autres termes, pour reprendre le point d'articulation proposé par Alain-Marie Bassy, cette série de dessins animaliers serait-elle destinée à "produire des

5. Voir le deuxième chapitre de la troisième partie: A. Gaillard, Fables, mythes, contes, L'esthétique de la fable et du fabuleux (I660-1724), 1996, p. 337-364.

6. Voir à ce sujet J. Bloch, art. cité, p. I38. Nous avons toutefois laissé de côté «La Chatte blanche» car, bien que ce conte soit représentatif de la question qui nous occupe — la métamorphose - , il n'a pas été illustré par Marillier.

7. Br. Bettelheim, Psychanalyse des contes de fées, 1979, p. 46I-466.

8. Voir le deuxième chapitre de la deuxième partie, intitulé "Les animaux»: A. Defrance, Les contes de fées et les nouvelles de Madame d'Aulnoy (I690-I698). L'imaginaire féminin à rebours de la tradition, 1998, p. II5-I54. Dans le cadre de cet article, nous ne pourrons toutefois traiter en profondeur, faute d'espace, des deux autres contes de Madame d'Aulnoy, illustrés par Marillier, qui renvoient plutôt à la catégorie de "l'animalité positive» : «La Biche au bois» (Cat. I2, CDF 03-3) qui appartient à la sous-catégorie de la "fiancée-animal »; de même que «Le Pigeon et la Colombe » (Cat. I4, $C D F$ 04-2), dans lequel les protagonistes métamorphosés optent définitivement pour la condition animale.

9. Nous suivons la numérotation du catalogue dressé par A. Belin dans : A. Zygel-Basso (dir.), Imager la Romancie. Dessins de Clément-Pierre Marillier pour Le Cabinet des fées et Les Voyages imaginaires (I785-I789), 2013, p. 453-458. Les dessins de Marillier pour Le Cabinet des fées (de même que pour les Voyages imaginaires) ont été mis en ligne par S. Lojkine sur la base de données UtpicturaI8 : < http://sites.univ-provence.fr/pictura/Presentation.php>.

IO. Voir à ce propos J. Rousset, Passages, échanges et transpositions, I990, p. I36. 
effets plus violents et plus assurés que ceux du discours ${ }^{\mathrm{II}}$ ", modifiant ainsi la trajectoire du texte, convoquant tant l'implicite que l'explicite?

Par ailleurs, il convient de replacer les planches qu’a proposées Marillier pour Le Cabinet des fées dans le contexte historique de l'illustration du genre. Comme l'a bien souligné A. Gaillard, déjà au XVII siècle, «l'adjonction d'illustrations, de devises ou de commentaires (de moralités et d'explications) transformait fables et contes en des sortes d'emblèmes, exhibant là encore l'aspect allégorique ou didactique des récits ${ }^{12} »$. Si Marillier propose des dessins qui s'inscrivent au cœur de considérations poétiques, esthétiques, culturelles et éthiques propres à la fin du XVIII ${ }^{\mathrm{e}}$ siècle - et donc sensiblement différentes de celles qui prévalaient au moment de la rédaction des contes - , il n'en reste pas moins que la "traduction ${ }^{13}$ " à laquelle il s'adonne en tant qu'illustrateur participe d'une structure qui existait un siècle auparavant : le texte se trouve au centre d'un dispositif comprenant minimalement image, titre et moralité. Ainsi, il convient d'aborder les contes de Madame d'Aulnoy que Marillier a illustrés dans Le Cabinet des fées en considérant en arrière-plan une structure semblable à celle qu'avait établie Perrault : «[...] une image liminaire [...], suivie du conte et d'une ou plusieurs "Moralités" ${ }^{14}$ ", à la différence près que l'illustration ne sera pas placée en vignette au début du conte, mais bien insérée en pleine page à l'endroit correspondant approximativement au texte de la séquence représentée, lui-même repris dans un cartouche situé au bas du dessin. Cet aspect est important dans la mesure où le choix (tant des contes illustrés que des épisodes représentés) a vraisemblablement été conditionné pour l'ensemble des volumes du Cabinet des fées par cette liste de légendes qui aurait été fournie à Marillier par l'éditeur ${ }^{15}$, afin qu'il les place dans les cartouches. Il sera donc intéressant de tenter de déterminer si l'illustrateur semble avoir parcouru vaguement le texte, ou encore si sa "traduction» reflète une appréciation plus subversive du contexte plutôt qu'un simple redoublement de la morale ${ }^{16}$ qui clôt les contes. Quoi qu'il en soit, il ne

II. A.-M. Bassy, art. cité, p. I53-I54.

I2. A. Gaillard, ouvr. cité, p. 233 [souligné dans le texte].

13. Pour de plus amples détails, on pourra consulter avec profit : A. Audy-Trottier et K. Gladu, "Le discours des éditeurs ou le rôle pédagogique de l'imagination : le Cabinet des fées et les Voyages imaginaires», dans A. Zygel-Basso (dir.), ouvr. cité, p. I25-I44.

I4. A. Gaillard, ouvr. cité, p. 235.

I5. Nous remercions A. Zygel-Basso pour nous avoir communiqué cette information. Bien que rien ne le prouve, il serait très étonnant que Marillier ait fait lui-même le choix (tant en ce qui a trait aux trois contes par volume à illustrer, qu'à celui des séquences à dessiner).

I6. Il s'agit en fait, dans les contes de Madame d'Aulnoy, d'une condensation en une seule moralité de ce qui constituait par exemple chez Perrault un redoublement des moralités : premièrement, 
s'agira pas tant de s'interroger sur le choix de la séquence représentée, que d'analyser de quelle manière Marillier a interprété le texte ou le contexte pour "traduire» sa vision des différents épisodes en lien avec la métamorphose animale.

\section{Métamorphose et animaux dans les illustrations du Cabinet des fées}

Il y a somme toute assez peu d'animaux représentés dans les dessins réalisés par Marillier pour Le Cabinet des fées, en regard de l'omniprésence de la gent animalière (réelle ou enchantée) animant la diégèse des contes, conformément à la "longue tradition narrative que l'on rencontre déjà dans les mythes antiques et les récits médiévaux ${ }^{17}$ » : seulement 36 des quelque I25 planches (28\%) présentent une espèce animale - nous incluons ici les hybrides tels les centaures et les créatures mythologiques tels les dragons - , accompagnant presque invariablement un ou plusieurs protagonistes à figure humaine (y compris fées, génies ou dieux). De plus, si l'on exclut une vingtaine d'illustrations où les animaux les plus conventionnels ne semblent avoir aucun rôle prépondérant dans le schéma actantiel, à tout le moins pour mériter le titre de héros animal - par exemple un cheval qui sert de monture (Cat. 75, "Mignonnette", $C D F$ 24-3) ou un dromadaire qui porte les provisions (Cat. II9, "Histoire de Ravie la résignée", $C D F$ 40-2) —, il ne reste guère qu'environ to \% des gravures qui proposent l'animal comme acteur principal ${ }^{18}$. Enfin, nous n'avons repéré que cinq planches pouvant être associées à la question de la métamorphose animale (moins de $5 \%$ de l'ensemble du Cabinet des fées); toutefois, hormis "Peau d'Âne» (Cat. 6, CDF OI-3), les quatre autres proviennent des contes de Madame d'Aulnoy, illustrant sans doute l'importance du thème chez l'auteure. En effet, rappelons que, pour des raisons essentiellement économiques, chacun des 4I volumes du Cabinet des fées ne comprend que trois gravures. Comme tous les contes de Madame d'Aulnoy (sauf «L'Île de la Félicité»), ainsi que leurs récits-cadres, occupent les volumes II à

une «transition entre le récit et la leçon à proprement parler, [qui] reprend des éléments de l'histoire», puis une seconde moralité de "portée plus générale» (A. Gaillard, ouvr. cité, p. 235).

17. V. Meunier, art. cité, p. 374.

18. Nous avons inclus dans ce décompte les deux planches présentant centaure ou centauresse, exemples emblématiques de la représentation de l'humanimalité dans les contes de fées (Cat. I8, La Tyrannie des Fées détruite, CDF 05-03 et Cat. 82, "Cinquième Veillée», CDF 27-I). Voir à ce sujet notre contribution : S. Paradis, «Signifier ou suggérer? Sobriété d'une faune fabuleuse", dans A. Zygel-Basso (dir.), ouvr. cité, p. 345-372, et spécialement p. 359-360. 
IV de l'anthologie, c'est donc dire que quatre des neuf contes illustrés convoquent la métamorphose animale. Il ne faut donc pas se fier à ce nombre relativement restreint, ou encore au fait que Marillier n'ait pas représenté certains autres contes emblématiques du phénomène de la métamorphose (par exemple "L'Oiseau bleu» ou "La Chatte blanche» de Madame d'Aulnoy dans les volumes II et IV, ou encore "La Belle et la Bête» de Madame de Villeneuve dans le volume XXVI) pour minimiser l'importance de ces planches dans l'économie de la poétique de la représentation qui se déploie dans la série illustrée. Il s'agira donc de s'intéresser à la manière dont Marillier représente la métamorphose animale, notamment au «moment» qu'il choisit d'illustrer en regard de l'épisode textuel où s'insère la planche.

Dans cette optique, il faut rappeler que la métamorphose animale, justifiée ou non, joue une triple fonction narrative : pénitence — châtiment qui n'est jamais définitif (sauf lorsqu'il s'agit de punir un méchant, ce qui n'est pas le cas en ce qui a trait aux dessins que nous analyserons plus en détails) —, expression métaphorique des rapports entre l'homme et la femme, et dualité mi-humaine, mi-animale qui conduit à une réflexion plus large sur la nature et la société. S’il arrive parfois que les animaux «interviennent pour seconder le héros à travers ses épreuves ou contre l'adversitér9» («La Belle aux cheveux d'or» de Madame d'Aulnoy) ou encore, selon une tradition empruntée aux fabulistes, qu'ils tiennent le rôle d'un être humain («Le Chat botté» de Perrault), Marillier semble plutôt avoir privilégié dans ses illustrations des séquences qui témoignent de la métamorphose temporaire du protagoniste humain. Par ailleurs, du point de vue du schéma actantiel, l'animal se révèle d'une polyvalence remarquable, offrant "à l'imaginaire humain de multiples ressources pour représenter ses fantasmes, pour incarner ses peurs et ses désirs ${ }^{20}{ }^{2}$. Les illustrations de Marillier qui renvoient au phénomène de la métamorphose seront donc un lieu privilégié d'expression du symbolique parallèle à celui que l'on peut explorer grâce à l'analyse textuelle, d'autant plus que l'exploitation de ce thème permettait aux auteurs ou illustrateurs de convoquer des «sujets difficilement recevables ou dicibles $[\ldots]$ : celui de l'androgyne, de travestissement, mais aussi de la souillure, de l'animalité ou encore du sabbat ${ }^{2 \mathrm{II}}{ }$.

L'analyse iconographique que nous proposons ne pourra évidemment pas faire l'économie de cette accélération temporelle qui sous-tend inexorablement tout passage d'une forme à une autre, depuis les Métamorphoses

19. V. Meunier, art. cité, p. 374.

20. A. Defrance, ouvr. cité, p. II6.

21. A. Gaillard, ouvr. cité, p. 340. 
d'Apulée, jusqu'aux contes de Madame d'Aulnoy que nous examinons ici, en passant par «Peau d'Âne» ou «Cendrillon». Cette interruption du continuum temporel qui surgit en un éclair pose évidemment un problème de taille pour l'illustrateur. En effet, s'il est possible de suggérer dans le texte la quasi, voire la complète simultanéité de deux états, si le «moment» de la transformation, sans être décrit, peut être énoncé sans évoquer aucun déroulement précis, on comprendra que les illustrations de Marillier - fort probablement générées, rappelons-le, par une légende redoublant un passage du texte mis en cartouche - ignorent le processus même (qu'il s'agisse du passage de l'humain à l'animal ou du retour à la forme humaine) qui, dans le texte, s'exprime de toute manière le plus souvent sans qu'il y ait vraiment trace d'un passage. Or, comme le souligne Aurélia Gaillard, «la vraie transformation n’a pas lieu lors de la métamorphose, qui [...] ne change pas la nature profonde des êtres, mais par la suite : c'est l'après-métamorphose, sa résorption, qui est le véritable acte de naissance $22 »$. Il s'agira alors de voir comment ces illustrations où les animaux accompagnent les héros humains traduisent des moments "of high emotion or dramatic confrontation ${ }^{23}$ " ("d'intense émotion ou d'affrontement spectaculaire»), autrement dit, révèlent une "dramatisation en images $[\ldots]$ où les scènes galantes avoisinent les scènes troublantes ${ }^{24}$ ». Nous essaierons donc d'établir si Marillier arrive à dépasser la simple paraphrase, s'il invite le lecteur à imaginer les péripéties sans se limiter à la représentation fidèle d'une scène, en somme s'il parvient à passer du statut d'illustrateur à celui de "conteur bis ${ }^{25}$ ", tout en conservant "l'expression de la vitesse et de la stupeur ${ }^{26}$ » qui s'avère un lieu commun du récit de métamorphose, et ce, même si le processus de la transformation — "figure pourtant topique de la fiction féerique et riche de possibilités iconographiques ${ }^{27} »-$ n'est jamais représenté. Chose certaine, nous pouvons anticiper et proposer d'emblée que Marillier ne tablera donc pas sur le côté spectaculaire $^{28}$ auquel lecteurs et cinéphiles d'aujourd'hui ont été habitués,

22. Ibid., p. 353 .

23. A. Lewis, Sensibility, reading and illustration. Spectacles and signs in Graffigny, Marivaux and Rousseau, 2009, p. I2I. Ma traduction.

24. C. Picaud, «L'illustration du conte de fées», dans O. Piffault (dir.), ouvr. cité, p. I6I.

25. Ibid., p. 158 .

26. A. Gaillard, ouvr. cité, p. 34I.

27. C. Martin, "Lillustration du conte de fées (1697-I789)", CAIEF, n 57, 2005, p. II9.

28. Pour de nombreux exemples de toiles, enluminures ou gravures qui ont exploité, depuis le Moyen Âge, le côté spectaculaire du processus de métamorphose, voir M. Warner, Fantastic Metamorphoses, Other Worlds. Ways of Telling the Self, 2002. Pour un aperçu de la variété des illus- 
qu'il s'agisse par exemple de l'illustration de Frédéric Clément pour «La Biche au bois ", qui représente la princesse Désirée en pleine mutation ${ }^{29}$, ou encore de cette scène magistrale dans le film La Belle et la Bête, produit par les studios Disney (I99I), où la métamorphose montre «la continuité entre la Bête et le Prince» alors que «la Bête tournoie dans les airs se transformant à vue pour devenir le Prince de façon si convaincante que la Belle peut vraiment lui dire : "Oui! c'est toi!" ${ }^{\circ}{ }^{\prime}$. En effet, si l'éditeur ou l'illustrateur avaient voulu insister sur la métamorphose en action dans les contes de Madame d'Aulnoy, ils auraient probablement jeté leur dévolu, dans le volume II du Cabinet des fées, sur "L'Oiseau bleu», "un de ces rares textes où le moment de la métamorphose est un peu développé ${ }^{31}$ ». Il ne faudrait cependant pas associer, peu s'en faut, cette relative sobriété à l'évacuation de toute "théâtralisation du fabuleux ${ }^{32}$ » qui s'affiche en amont et en aval du retour à la forme humaine des fiancés-animaux, comme nous le verrons dans les deux cas de figure qui suivent.

\section{Le fiancé-animal métamorphosé : «Serpentin Vert» entre l'explicite et le codage galant}

Dans le premier cas de figure que nous étudierons plus attentivement, le prince (Serpentin) a fait les frais d'une mauvaise fée (Magotine) — agent de la métamorphose - et subit l'animalité qui devient l'épreuve qu'il faut affronter avant de retrouver une forme humaine. Parallèlement, peu de temps après sa naissance, une des deux filles jumelles de la reine est transformée en "la plus laide créature du monde ${ }^{33}$ » $(C D F$, III, p. I77) encore ici par la même «barbare petite fée» ( $C D F$, III, p. I76) Magotine, parce que celle-ci n'avait pas été invitée, à l'instar de douze de ses consœurs, pour

trations de contes de fées en général, du XVII ${ }^{\mathrm{e}}$ siècle à nos jours, voir $\mathrm{C}$. Picaud et $\mathrm{O}$. Piffault, Contes de fées en images. Entre peur et enchantement, 2013.

29. Madame d'Aulnoy, L'oiseau bleu et autres contes, I991.

30. L. Cheilan, «La Bête et le Prince dans quelques versions illustrées ou versions en images de La Belle et la Bête», dans J. Chiron et C. Seth (dir.), Marie Leprince de Beaumont. De l'éducation des filles à La Belle et la Bête, 20I3, p. I84.

3I. A. Gaillard, ouvr. cité, p. 34I.

32. Ibid., p. 239.

33. Les extraits des contes de Madame d'Aulnoy que nous citons (en modernisant l'orthographe) proviennent des volumes III et IV de l'édition suivante : C.-J. de Mayer (éd.), Le Cabinet des fées ou Collection des contes de fées, et autres contes merveilleux ornés de figures, Genève, Barde, Manget $\&$ Cie, à Paris chez Cuchet, 1785 . Désormais, nous inscrirons les renvois à cette édition dans le texte, entre parenthèses, par l'acronyme $C D F$, suivi du numéro de volume en chiffres romains, puis du numéro de page(s) où se retrouve l'extrait cité. 
douer les nouveau-nées. Les deux histoires se rejoignent à mi-parcours du conte pour atteindre un climax renvoyant à la moralité finale : la désobéissance de la princesse Laidronette — si «affreuse» qu'il était «impossible de la regarder" $(C D F$, III, p. I78) —, qui succombe au topos de la "curiosité fatale» (CDF, III, p. 20I), ce qui aura comme conséquence de condamner son époux le roi Serpentin à recommencer sa pénitence, lui qui avait déjà purgé cinq des sept ans pendant lesquels il avait été métamorphosé.

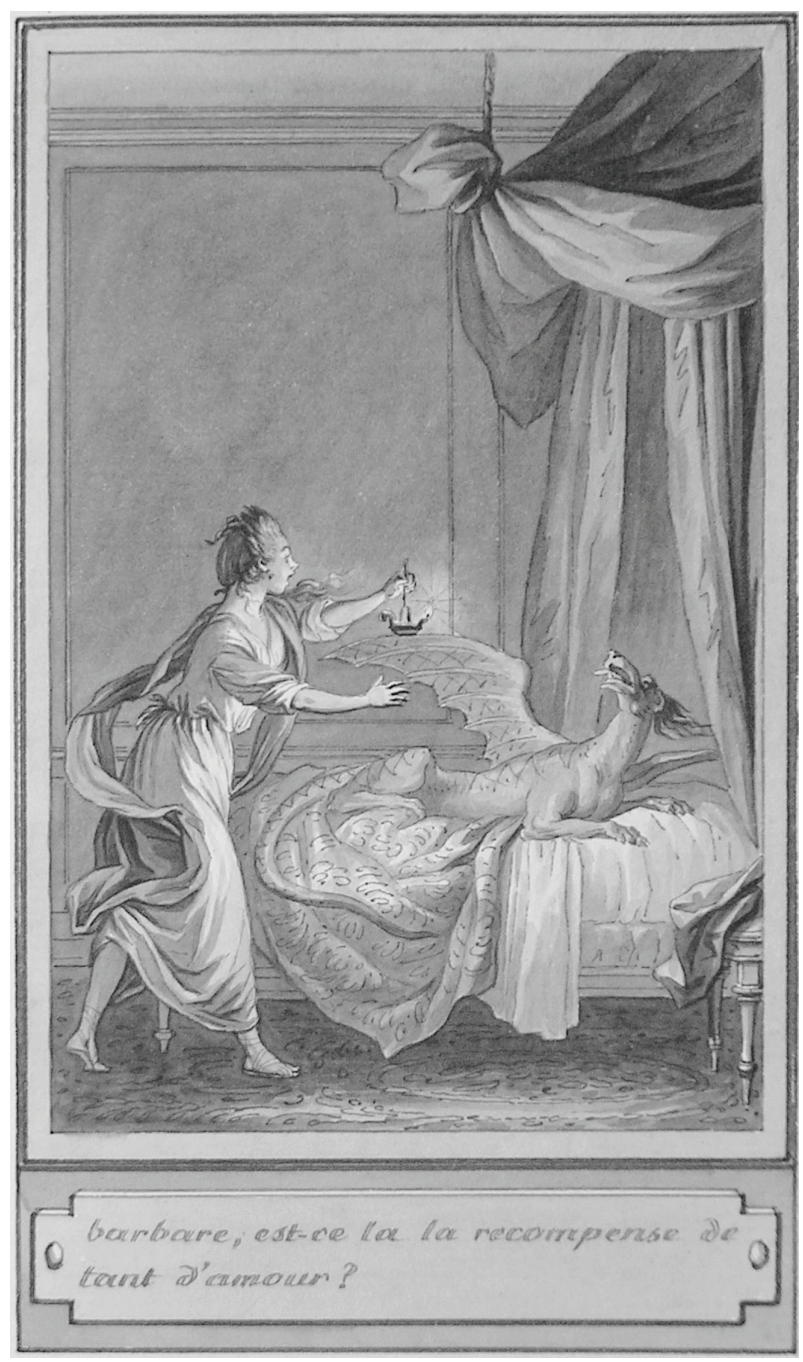

Fig. I. — «Serpentin Vert» tiré du Cabinet des fées chez Manget \& Cie, Genève, I785.

Collection privée. 
Il s'agit précisément de la scène que Marillier a illustrée (Cat. II, «Serpentin vert ", $C D F$ 03-2), selon un point de vue omniprésent dans les gravures du Cabinet des fées : «la théâtralisation des postures en fonction du "moment" choisi» qui révèle souvent "des motifs tels que l'effraction, la séduction et le dévoilement " ${ }^{34}$, indiquant "l'instant à peine saisissable où l'univers bascule, où la chute est déjà présente sans avoir pourtant commencé35». Que ce parti pris pour la théâtralisation d'un moment qui précède ici la fuite de Serpentin soit la résultante d'une recherche d'intensité dramatique, ou, comme le propose Christophe Martin, la répétition d'un motif qui suggère des «menaces premières" ou des "craintes archaïques ${ }^{36}$ qui renverraient à une sorte de culture inconsciente de l'enfance, le constat est le même : Marillier joue à la fois sur deux tableaux. D'une part, il signifie en renvoyant le lecteur à des schémas anthropologiques de l'imaginaire reliés à cette naïveté, à cette violence enfouie dans l'enfance, et, d'autre part, il suggère également, en créant "un effet de distance» où «le cadre de l'image et la légende des gravures offrent le simulacre d'un tableau ${ }^{37}$. Inutile de trop insister ici sur les liens entre animalité et enfance, inscrits au cœur même de la métamorphose animale qui, dans la majorité des cas, renvoie au contexte pré-babélien des animaux humains parlants, permettant à la fois de gommer les différences entre humanité et animalité sans éveiller la censure, et d'établir clairement «l'équivalence entre enfance et animalité ${ }^{38}$ ".

En effet, comme dans la majorité des cas de métamorphose animale, Serpentin a conservé la capacité de raisonner, de même que la parole ${ }^{39}$ grâce à laquelle il a charmé son épouse, et il ressent le corps qui lui a été attribué comme une prison, illustrant ainsi parfaitement «le passage d'une âme dans un autre corps : une métempsychose ${ }^{40}$ " où l'humain devenu animal conserve son affect d'humain. Certes, la fonction didactique ou morale semble bien servie ici par le dessin — plus l'apparence extérieure

34. C. Martin, «L'émergence d'un nouvel objet de recherches : le roman illustré au XviII ${ }^{\mathrm{e}}$ siècle», dans P. Stewart et M. Delon (dir.), Le second triomphe du roman du XVIII esiecle, 2009, p. 202.

35. C. Martin, "Dangereux Suppléments». Lillustration du roman en France au XVIII siècle, 2005, p. XII.

36. C. Martin, «L'illustration du conte de fées (1697-1789)», art. cité, p. I23.

37. Ibid., p. 124 .

38. A. Gaillard, ouvr. cité, p. 324.

39. Voir à ce sujet M. Slater, "Les animaux parlants dans les Contes de fées de $\mathrm{M}^{\mathrm{me}} \mathrm{d}$ 'Aulnoy", dans J. Perrot (dir.), Tricentenaire Charles Perrault. Les grands contes du XVII siècle et leur fortune littéraire, 1998, p. I57-I64.

40. A. Gaillard, ouvr. cité, p. 347. 
est méprisable, plus dure est l'épreuve à supporter ${ }^{41}$ — chez ce qui nous semble un dragon "symbole du mal», "gardien des trésors cachés, et comme tel l'adversaire qui doit être vaincu pour y avoir accès ${ }^{42}$. En effet, cette iconographie qui fait partie de figures «récurrentes et stéréotypées, invitant le lecteur à retrouver des images toujours déjà connues ${ }^{43}$ " met en lumière une des fonctions de l'illustration jouant sur l'exploitation du lieu commun qui sert en quelque sorte ici à «éveiller l'imagination du lecteur, et à offrir une caution culturelle au spectacle en suscitant dans sa mémoire des réminiscences de ses lectures naturalistes ${ }^{44}$ ». Mais le Serpentin Vert dessiné par Marillier, à y regarder de plus près, s'avère somme toute plutôt banal ${ }^{45}$, ayant notamment la taille plutôt menue pour un supposé "gros Serpent vert» $(C D F$, III, p. I8I), si on la compare à celle de la princesse. Au-delà des impondérables liés au format — il faut bien faire entrer dans le cadre les protagonistes que l'on veut représenter avec un minimum de détails (ne serait-ce que pour pouvoir déceler les émotions telles que la terreur ou la pitié sur le visage) —, se profile peut-être également la volonté du dessinateur de marquer ses créations d'un certain anthropocentrisme latent, magnifiant par le fait même la représentation de la puissance des héros humains ${ }^{46}$. Ce qui nous pose problème, à nous lecteurs modernes, et qui constitue un élément de surprise se superposant à la stupeur de la princesse Laidronette au moment où elle rompt son serment et à la lueur d'une lampe entrevoit l'hideuse conformation de son mari, ressort de l'onomastique : le nom de l'animal suggère le traditionnel reptile qui força Ève à croquer la pomme, alors que le dessin propose plutôt un dragon en miniature. Surdéterminant la dichotomie réel / fiction, on remarquera dans cette gravure que le serpent-dragon arbore une tête toute canine. Peut-être s'agit-il d'un exemple de ce paradoxe auquel fait référence Christophe Martin lorsqu'il rappelle que l'illustration du conte de fées, qui semble aller de soi pour nous, est une pratique essentiellement postérieure au XVIII ${ }^{\mathrm{e}}$ siècle $^{47}$. Aussi notre expérience de lecteurs modernes accoutumés

4I. V. Meunier, art. cité, p. 376.

42. J. Chevalier et A. Gheerbrant, «Dragon», Dictionnaire des symboles, 1982, p. 366.

43. C. Martin, "L'illustration du conte de fées (I697-I789)", art. cité, p. II7.

44. M. Levacher, «Les lieux communs dans l'Histoire naturelle de Buffon : rhétorique judiciaire, rivalité dans la récriture et compromis épistémologique", Dix-huitième siècle, nº 42, 20IO, p. 37.

45. Nous remercions A. Zygel-Basso pour nous avoir souligné que le motif du dragon est très à la mode dans le papier peint pendant la dernière décennie du XVIII ${ }^{\mathrm{e}}$ siècle, ce qui pourrait avoir influencé ici la représentation adoptée par Marillier.

46. Sur le problème spécifique des proportions dans les dessins animaliers de Marillier, nous renvoyons à notre article : S. Paradis, art. cité, spécialement p. 352-358.

47. C. Martin, «L'illustration du conte de fées (I697-I789) », art. cité, p. II3. 
à voir des dragons à tête de dragon dans les contes (imprimés ou portés à l'écran) ne doit-elle pas être contaminée par cette illusion rétrospective : au XVIII ${ }^{\mathrm{e}}$ siècle, le dragon était le plus souvent cynocéphale, et serpents et dragons se confondaient. Précisons que Marillier respecte ici non seulement la lettre du texte - la fée Magotine a en effet métamorphosé le prince en «gros Serpent vert» $(C D F$, III, p. I8I), caractérisé comme un "horrible monstre" aux "ailes verdâtres", aux "griffes d'ivoire" et à la tête "hérissée de longs crins" (CDF, III, p. I8I-I83), détails bien identifiables sur la gravure -, mais il répond aussi à l'horizon d'attente des lecteurs de l'époque pour lesquels le dragon est un "animal fabuleux que l'on s'est représenté sous la forme d'un serpent avec des ailes et des pieds ${ }^{48} »$. Il est loin d'être certain que le public des Lumières se soit complètement démarqué d'une époque pas si lointaine où ses prédécesseurs de la Renaissance reconnaissaient, par l'image, l'animal décrit dans le texte, parce que "l'illustration évoqu[ait] non pas l'animal réel mais plutôt la représentation mentale qu'en a[vait] le lecteur ${ }^{49}$ ». Ainsi s'opposent aux animaux familiers ou domestiques, que l'on côtoie quotidiennement, les animaux lointains, qui peuvent l'être «soit parce qu'ils sont difficiles à observer, soit parce qu'ils vivent dans des contrées éloignées, soit encore parce qu'ils vivent dans l'imaginaire collectif ${ }^{5 \circ} "$, bien ancrés comme «lieu[x] commun $[s]^{\mathrm{sI}} »$. Il reste que l'implicite de cette planche réside selon nous ailleurs. En effet, les animaux issus de la métamorphose, par l'humanité qui leur est conférée ou "par les prodiges qu'ils accomplissent ou la terreur qu'ils suscitent", servent en quelque sorte ainsi de "projections affectives qui permettent au lecteur de mieux comprendre le monde et de combler son besoin de

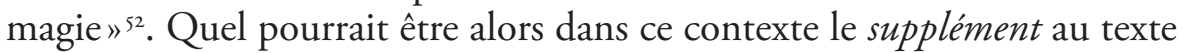
suggéré par cette planche illustrant le "Serpentin Vert», investie par le principe de théâtralisation reposant ici sur les motifs de l'effraction et de la séduction? Afin d'y répondre, il importe de replacer l'épisode illustré dans la séquence du conte, en regard de la moralité finale, et de regarder peut-être non pas du côté de l'agent ou du sujet de la métamorphose, mais bien de celui de la pas si laide Laidronette...

En effet, l'image a de quoi étonner de prime abord : Laidronette est incidemment fort peu ravagée pour une princesse à qui la fée Magotine

48. L.-J.-M. Daubenton, «Dragon, draco, (Hist. natur. Zoolog.)", Encyclopédie ou Dictionnaire raisonné des sciences, arts et des métiers, 1966, t. V, p. 104.

49. L. Pinon, Livres de zoologie de la Renaissance: une anthologie (I450-I700), 1995, p. 24.

50. Ibid., p. 3I.

5I. Sur les liens entre animal et «lieu commun» rhétorique, voir M. Levacher, art. cité, p. 35-54. Le même thème est traité par l'auteure dans Buffon et ses lecteurs, 20II, p. 55-85.

52. V. Meunier, art. cité, p. 377. 
est censée avoir ôté, peu de temps après la naissance, toute beauté pour la rendre "affreuse" ( $C D F$, III, p. 209), à tel point qu'elle déplore, quelque temps avant d'accepter d'épouser le « roi invisible» (CDF, III, p. 196), être devenue «la plus laide personne du monde» (CDF, III, p. 194). C'est à se demander si Marillier n'aurait pas sauté sur l'occasion de recycler une scène ou un dispositif mythologique archi-connu en parcourant vaguement le conte, sans respecter exactement le contexte ni prendre en compte le fait que Laidronette n'a pas encore bu «l'eau de discrétion" ( $C D F$, III, p. 210) qui l'embellira, et qu'elle n'a pas encore été transfigurée en reine Discrète. Cela dit, au-delà de l'apparence plutôt banale de Serpentin et de l'incongruité du faciès de Laidronette, on peut se demander en quoi cette gravure "prolonge $[\ldots]$ moins le texte qu'elle ne le transgresse ${ }^{53}$ ». Si l'on accepte qu'il puisse exister de multiples réseaux de signification, l'illustration pourrait donc simuler et dissimuler tout à la fois, et s'avérer un outil efficace de transgression, qui viendrait épouser en quelque sorte une des caractéristiques principales de l'écriture de Madame d'Aulnoy : la subversion, "mot-clé, véritable sésame de l'œuvre ${ }^{54}$ " selon Marie-Agnès Thirard. Comme aucune image n'est neutre, mais qu'elle opère au sein d'un réseau de présomptions idéologiques relatives à l'ordre naturel ou social et que son effet dépend aussi de conventions esthétiques inhérentes à l'épistémologie du langage de la représentation qui gouverne ce qu'elle doit diress, on pourrait alors proposer ici que la gravure satisfait le goût du lecteur en simulant le respect de la norme, tout en appelant un codage galant où "l'image constitue en système les procédés ou les feintes qui lui permettent d'enfreindre l'interdit sexuel ${ }^{56} »$. Ainsi, la complicité et les connivences avec le lecteur se superposeraient aux relations pédagogiques véhiculées par l'image — le renvoi à la moralité finale —, et viseraient aussi le plaisir individuel, supportant ainsi la thèse de C. Martin qui propose d'interpréter les illustrations en terme de "suppléments ${ }^{57}$ " au texte. Conséquemment, l'illustration servirait ainsi à "produire des effets plus violents et plus assurés que ceux du discours" afin de "toucher la

53. A.-M. Bassy, art. cité, p. I6I.

54. M.-A. Thirard, Les contes de fées de Madame d'Aulnoy, une écriture de la subversion, 1994, p. 582. Voir également, à ce propos, J. Mainil, Madame d'Aulnoy et le rire des fées : essai sur la subversion féerique et le merveilleux comique sous l'Ancien Régime, $200 \mathrm{I}$.

55. Voir, à ce propos, A. Potts, "Natural order and the call of the wild: the politics of animal picturing", The Oxford Journal, vol. I3, n I, I990, p. I2.

56. A.-M. Bassy, art. cité, p. I6I.

57. C. Martin, «L'émergence d'un nouvel objet de recherches [...] », art. cité, p. 202. 
sensibilité individuelle, de stimuler l'imaginaire de chaque lecteur ${ }^{58}$ ». Le thème de la surprise ou de la prise amoureuse dans la chambre à coucher, topos omniprésent de ce codage galant, se retrouve dans plusieurs gravures contemporaines où sont représentées des postures paroxystiques découlant de l'expression de la surprise imprégnée de sensualité, traduisant par exemple la pâmoison ou le travestissement. Pourrait-on alors aller jusqu'à affirmer, en suivant A.-M. Bassy, qu'ici l'image "sollicite la sensibilité, l'affectivité, voire la sexualités9 »? Selon Jeanne Bloch, l'animalité permettrait précisément de suggérer la sexualité, sans l'énoncer explicitement, et l'affreux Serpentin représenté sur la gravure serait à considérer comme "un symbole phallique clair ${ }^{60}$ " : l'adjectif hérissé caractérisant la crinière serait ainsi "porteur d'une connotation sexuelle en ce qu'il suggère de manière évidente l'érection masculine ${ }^{61}$ ", tout comme, ajouterions-nous, le parallélisme du museau, des ailes et de la queue. Le dessin représentant Laidronette et Serpentin serait donc peut-être, à l'image des estampes galantes, plus salace qu'il n'y paraît ${ }^{62}$, agissant à titre de métonymie, à l'instar de l'animal domestique, du jeune serviteur, de la carafe à col étroit, du verre d'eau débordant, de l'éponge ou de la chandelle. Malgré sa relative sobriété, la planche vise ici peut-être à "projeter l'imaginaire du lecteur vers un au-delà du texte», en faisant "constamment appel aux pouvoirs affectifs ou érotiques de l'image», confirmant ainsi «le pouvoir d'emprise de l'image sur le lecteur [...], qu'elle conforte les mécanismes de l'illusion romanesque, ou qu'elle incite au contraire à prendre ses distances à l'égard de la fiction " ${ }^{63}$. Marillier, consciemment ou non, se retrouverait donc à répéter par le biais de l'image le même processus subversif qui caractérisait l'écriture de Madame d'Aulnoy, appelant cette même connivence culturelle qui incite le lecteur initié à dépasser le monde des apparences.

L'érotisme et la sexualité implicites de la gravure renvoient par ailleurs aussi à la référence explicite au mythe de Psyché, soulignée à gros traits dans le texte de ce conte avec lequel il présente de singulières analogies : «[...] une jeune fille prend pour époux un invisible. Piquée de curiosité

58. A.-M. Bassy, art. cité, p. I53. Voir également à ce sujet N. Ferrand, Livres vus, livres lus : une traversée du roman illustré des Lumières, 2009.

59. A.-M. Bassy, art. cité, p. I53.

6o. J. Bloch, art. cité, p. I27.

6I. Ibid., p. I28 [nous soulignons].

62. Voir à ce sujet A. Guillerm, "Le système de l'iconographie galante», Dix-huitième siècle, $\mathrm{n}^{\circ}$ I2, I980, p. I83-184, qui situe l'apogée de l'estampe galante dans les années I770.

63. C. Martin, «L'émergence d'un nouvel objet de recherches [...] ", art. cité, p. 202. 
[...], elle s'approche de lui la nuit et l'éclaire de sa lampe. L'époux s'enfuit, et la jeune fille est plongée dans de terribles malheurs. Elle doit franchir plusieurs étapes avant de retrouver son époux et le bonheur ${ }^{64} »$. De plus, le livre que remet une pagode à Laidronette — «l'histoire de Psyché, qu'un auteur des plus à la mode venait de mettre en beau langage» (CDF, III, p. 198) — renvoie évidemment aux Amours de Psyché et de Cupidon, texte de La Fontaine publié en I669. Cependant Madame d'Aulnoy fait preuve d'originalité et s'écarte du mythe tel qu'il a été présenté par Apulée dans L'Áne d'or et repris par La Fontaine : Laidronette s'est vue condamnée à incarner la plus laide créature du monde, alors que Psyché était la plus belle personne de l'univers. Dans le cas où Marillier se serait contenté de parcourir négligemment le texte du conte, n’aurait-il pas eu cette image à l'esprit? De plus, dans le mythe, Psyché, qui a accepté d'épouser un monstre, découvre le dieu Amour dans son lit nuptial ; à l'inverse, Laidronette surprend « au lieu du tendre amour, blond, blanc, jeune et tout aimable, [...] l'affreux Serpentin Vert aux longs crins hérissés» (CDF, III, p. 20I-202). Comme l'a souligné A. Defrance, cette inversion est essentielle car elle conduit au "refus de la monstruosité» et à la "peur de voir et d'être vue» ${ }^{65}$, aspects importants de la métamorphose, qui gravitent autour de la relation à autrui. En ce qui a trait à la monstruosité, nous avons déjà insisté sur le fait que le texte présente un monstre dont la vue déclenche chez Laidronette une "frayeur épouvantable» ( $C D F$, III, p. I84), et ce bien avant la scène illustrée par Marillier, alors qu'elle croise Serpentin à la suite d'un naufrage. Par la suite, ne se doutant pas que son époux invisible est en l'occurrence le monstre qui lui a inspiré tant de répulsion, elle se laisse petit à petit gagner par le désir de le voir. Malgré les Aventures de Psyché qu'elle lit et relit pour "être en garde sur tout ce qu'on lui dirait» (CDF, III, p. 199), c'est finalement sur l'insistance de sa mère et de sa sœur qu'elle succombe sans pouvoir se garder «de ne pas imiter sa devancière Psyché» (CDF, III, p. 20I). Toujours selon A. Defrance, la leçon que l'on peut tirer de la suite des aventures de Laidronette et de Serpentin est que Madame d'Aulnoy prêcherait ainsi pour «une conduite indépendante de toute directive émanant de l'extérieur» qui rejoint une interprétation croisée avec la psychanalyse :

Madame d'Aulnoy présente donc une héroïne qui a intériorisé une représentation qui n'existait, dans les textes antérieurs, que dans le discours de l'autre. Cette intériorisation engendre une peur, un sentiment de répulsion, symptômes d'un refoulement.

64. A. Defrance, ouvr. cité, p. 239.

65. Ibid., p. 240. 
Toute sa conduite illustrera une trajectoire de libération par rapport à ses inhibitions [...]. Quand elle suit le modèle forgé par le discours de l'autre, elle se retrouve face au serpent hideux, manifestation symbolique des peurs antérieures qu'elle avait presque réussi à vaincre ${ }^{66}$.

Peur de la monstruosité, héroïne en position de révolte qui abhorre sa propre image et celle de Serpentin, préexistence du mythe de Psyché agissant comme un savoir aliénant, difficulté de vaincre la répulsion engendrée par le sexe opposé, toutes ces caractéristiques semblent se fondre aussi dans la planche dessinée par Marillier. Et ce même si, de prime abord, le dessin ne semble pas différer de la norme inhérente à l'illustration du conte merveilleux à la fin du XviII ${ }^{\mathrm{e}}$ siècle, et relever d'une relative pauvreté $^{67}$, voire d'une "remarquable sobriété ${ }^{68}$ ». En effet, le texte du cartouche insiste sur la cruauté de l'épouse qualifiée de «barbare», redoublant en quelque sorte la moralité explicite finale qui signifie, au premier degré, et met en garde contre le potentiel destructeur de la curiosité féminine :

Souvent un désir curieux

Est la source des maux les plus épouvantables :

Sur un secret qui doit nous rendre misérables,

Pourquoi vouloir ouvrir les yeux?

Le beau sexe a surtout cette ardeur si cruelle. (CDF, III, p. 227)

Mais en esthétisant la représentation, en y inscrivant un codage galant, en diminuant la proportion du monstre et, surtout, en désenlaidissant (volontairement par anticipation, ou par négligence) Laidronette, Marillier trouve peut-être aussi à rejoindre le potentiel subversif du texte de Madame d'Aulnoy. En effet, Laidronette est présentée dans ce conte, à la différence de Psyché, en position de révolte par rapport à sa propre image et à celle de Serpentin, dont la rencontre bien avant le mariage symboliserait «la difficulté à vaincre la répulsion causée par le sexe opposé ${ }^{69}{ }$. C'est, symboliquement, après s'être libérée de la parole familiale (en particulier de la mère, figurée par Magotine) et des mythes ancestraux qu'elle revêtira l'apparence de la reine Discrète et provoquera la "déserpentination» du roi de la Pagodie. C'est un peu comme si l'illustrateur avait deviné le message de l'auteure : tout comme celle-ci se joue du modèle mythologique destructeur, il suggère en parallèle «qu'il est possible et préférable de n'en

66. Ibid., p. 246-247.

67. Voir Ph. Stewart, «Images de ce qui ne fut jamais», dans R. Jomand-Baudry et J.-Fr. Perrin (dir.), Le conte merveilleux au XVIII siècle. Une poétique expérimentale, 2002, p. I4.

68. C. Martin, «L'illustration du conte de fées (I697-I789)», art. cité, p. III.

69. A. Defrance, ouvr. cité, p. 247. 
pas subir l'effet paralysant ${ }^{70}$ ", sa reine Discrète anticipée laissant entrevoir qu'il est possible de ne pas succomber au destin imposé par la méchante Magotine et de transgresser le tabou sexuel.

Le Prince "démarcassiné» : érotisation et esthétisation de l'humanimalité

Toutefois, aucun codage implicite ne se laisse percevoir dans la planche illustrant "Le Prince Marcassin», qui représente à nouveau un fiancéanimal destiné au mariage avec une héroïne humaine.

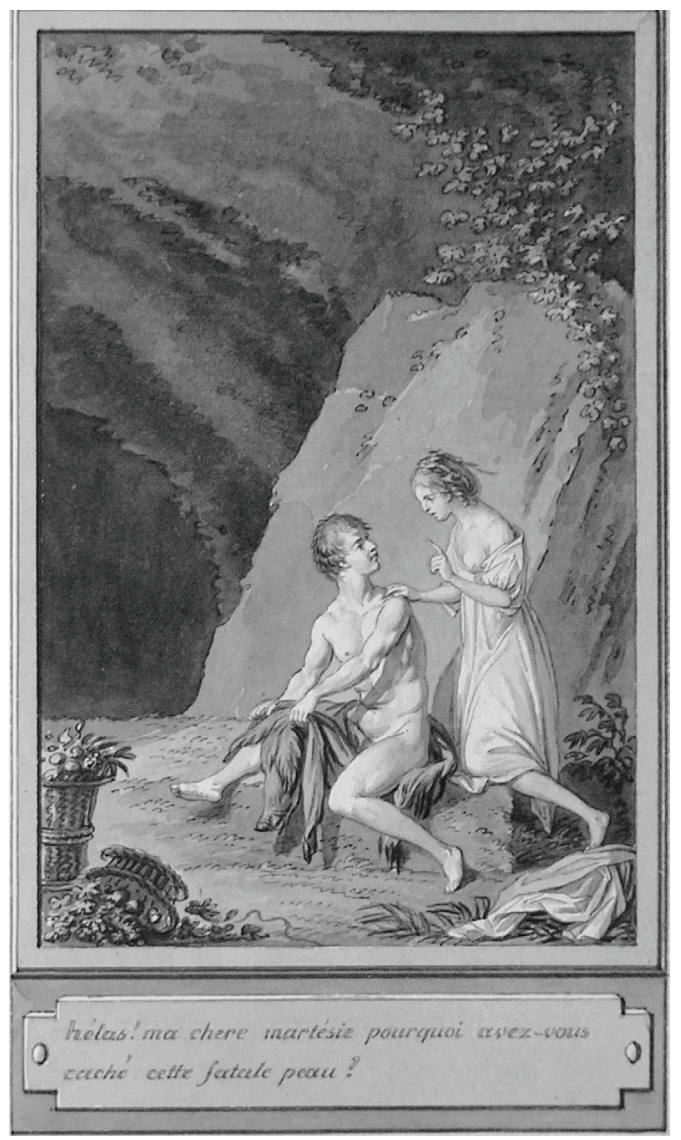

Fig. 2. — «Le Prince Marcassin» tiré du Cabinet des fées chez Manget \& Cie, Genève, 1785.

Collection privée.

7o. Ibid., p. 247. 
Le héros dépend de l'amour salvateur d'une femme pour pouvoir retrouver sa forme humaine et, tout comme dans "Serpentin Vert», le texte ne révèle pas les raisons ayant conduit ici la cadette de trois fées à condamner le Prince, avant même sa naissance, à «être Marcassin, jusqu'à ce qu'il ait épousé trois femmes, et que la troisième trouve sa peau de sanglier» (CDF, IV, p. 370). À la différence de "Serpentin Vert» toutefois, le texte insiste sur la cruauté de Marcassin : le narrateur "n'épargne aucun détail pour souligner à quel point la monstruosité est intolérable et fait naître chez les personnages féminins qui seront confrontés à elle la peur de la dévoration et la menace du viol ${ }^{71}$ ", exposant ainsi avec acuité l'animalité négative qui sous-tend la métamorphose. De plus, le mariage n'attendra pas le bon vouloir des trois sœurs convoitées successivement par le Prince Marcassin : la première, Ismène, ne pourra échapper que par le suicide au désir masculin faisant peser sur elle menace de dévoration et promesse de viol; la seconde, Zélonide, verra Marcassin se jeter sur elle et lui enfoncer "ses grandes défenses dans la gorge" $(C D F, \mathrm{IV}$, p. 352); seule la sœur cadette, Marthésie, sera courtisée avec douceur. De manière plutôt étonnante, le retour à la nature provoque un adoucissement de l'animalité, même si Marcassin finit par séquestrer Marthésie dans une caverne jusqu'à ce qu'elle accepte de l'épouser, contrairement à ce qu'il lui avait naguère promis. Madame d'Aulnoy opère ici un renversement homme / animal pour expliquer la conduite de Marcassin, en plaçant «nettement l'animal du côté du positif et l'homme de celui du négatif ${ }^{72} »$ :

Comment pourrai-je me fier à vos paroles, lui dit-elle, puisque vous manquez à la première que vous m'avez donnée? Il faut bien, lui dit-il en souriant [...], qu'il y ait un peu de l'homme mêlé avec le sanglier; ce défaut de parole que vous me reprochez, cette petite finesse où je ménage mes intérêts, c'est justement l'homme qui agit; car pour parler sans façon, les animaux ont plus d'honneur entr'eux que les hommes. $(C D F, \mathrm{IV}, \mathrm{p} .364)$

Nonobstant ce reproche, Marthésie finit par épouser le prince, acceptant l'animalité qui graduellement devient de moins en moins repoussante, voire de plus en plus désirable, au cours des six mois qu'elle passe dans la grotte avec le sanglier dont la "douceur extrême» la conduit à lui témoigner «beaucoup de tendresse», si bien que Marcassin la lui rend ensuite en la chérissant «avec la dernière passion" (CDF, IV, p. 368). Il importe

7I. Ibid., p. I20.

72. Ibid., p. I22. 
de noter toutefois que cette modification se produit en miroir, au fur et à mesure que Marthésie se rend compte que «la hure [de Marcassin] était faite comme la tête d'un homme» ( $C D F, I V, p .366)$, jusqu'à ce qu'elle lui avoue : "Croyez-vous [...] que je ne sache pas que vous cédez toutes les nuits votre place à un homme?» (CDF, IV, p. 366) Selon A. Defrance, ce discours rejoindrait l'interprétation psychanalytique traditionnelle selon laquelle "la répulsion première de la femme face à l'animalité traduit son angoisse du sexuel» et suggérerait qu'une fois consommée, la relation "perd l'aspect agressif et repoussant que la femme avait fantasmé » ${ }^{73}$. En effet, sans que le texte n'appuie nullement sur la description des ébats amoureux, on apprend que Marthésie est "grosse», et qu' elle ressent "une affliction sans pareille» à l'idée que "la race marcassine [va] se perpétuer» (CDF, IV, p. 368). Puis, pendant la nuit, alors qu'elle entend chuchoter "le bon Marcassin qui priait une personne de lui être moins rigoureuse", Marthésie trouve sous ses pieds «la peau d'un sanglier» (CDF, IV, p. 369), et la cache en attendant l'aurore.

C'est précisément à la suite de cet épisode que se produit la démarcassination complète du prince, instantané qu'a reproduit Marillier depuis le passage suivant inséré dans le cartouche : "Hélas! ma chère Marthésie, [...] pourquoi avez-vous caché cette fatale peau?» (CDF, IV, p. 373) Tout d'abord, l'expression de dépit qui se lit sur le visage du prince traduit fidèlement le texte qui précède immédiatement le moment représenté, tandis que Marcassin tente désespérément de remettre la peau de sanglier «devenue si petite, qu'il n'y avait pas de quoi couvrir une de ses jambes. [...] Il était bien triste et déplorait son malheur; car il craignait [...] que la fée qui l'avait si bien marcassiné, ne vint la lui remettre pour longtemps» (CDF, IV, p. 373). Par ailleurs, Marillier semble encore une fois soit avoir négligé le contexte, soit avoir voulu suggérer une atmosphère qui n'est certes pas transparente à la lecture. En effet, rien sur la gravure ne laisse présumer la possible grossesse de Marthésie, mais la séquence se trouve plutôt érotisée, tant par la posture du prince tentant nonchalamment d'enfiler sa peau trop petite - est-ce un hasard si le groin semble se superposer à ce que la feuille cachait chez Adam voire le prolonger? que par celle de Marthésie, le sein droit explicitement dévoilé. En effet, cette érotisation de la scène est à chercher ailleurs que dans l'explicite du texte et se retrouve également dans un nombre impressionnant de dessins

73. Ibid., p. I23. 
réalisés par Marillier pour Le Cabinet des fées ${ }^{74}$. Cette «traduction" semble d'une part s'inscrire tout à fait dans une perspective plus globale de la série illustrée et, comme l'ont noté Andréane Audy-Trottier et Kim Gladu, cela sert davantage à susciter l'émotion du lecteur qu'à décrire des mondes enchantés, tout en visant à transmettre une instruction morale par le canal de la sensibilité :

L'image propose une première lecture du texte et, dès lors, en mettant en évidence non pas le merveilleux des récits, mais leur potentiel pathétique et donc pédagogique, les dessins de Marillier participent à ce goût général où l'on fait servir à l'imagination un but moral et non plus seulement d'agrément ${ }^{75}$.

Cette morale a peut-être encore une fois à voir avec l'implicite. En effet, la réplique qui traduit le dépit du Prince «démarcassiné», reproduite dans le cartouche, est censée survenir «[p]endant» (CDF, IV, p. 373) que Marthésie adresse des compliments aux fées pour les remercier d'avoir si bien pourvu à leurs besoins au cours des six derniers mois passés dans la grotte. Or, force est de constater que l'héroïne ne semble pas louanger les fées, mais bien plutôt s'adresser à Marcassin, sans qu'il soit vraiment possible de déterminer si l'index levé suggère l'interjection, la concession ou l'opposition. De plus, si on prend la peine de retourner au texte de Madame d'Aulnoy, et de consulter la séquence qui suit immédiatement la réplique reproduite dans le cartouche, on constate que Marthésie se met à pleurer, en réponse à l'inquiétude du Prince qui croit que le rétrécissement de la peau de sanglier est une punition résultant de la colère des fées. La gravure ne représente pas clairement cette émotion. En fait le narrateur intervient à ce moment pour souligner l'incongruité de cette réaction : «[...] c'était là un sujet d'affliction bien singulier de pleurer, parce qu'il ne pouvait plus devenir Marcassin.» (CDF, IV, p. 373) Le retour à la forme humaine survient dans un climat de tristesse et de consternation partagé par les deux héros, et il est ressenti plus comme un méfait (le vol de la peau) que, comme habituellement dans les autres contes, la réparation d'un méfait. Cette scène paradoxale se trouve subvertie par le retour inattendu des fées qui, par une pirouette morale ayant trait à la réalité et aux illusions produites par l'imagination, restaurent un climat d'allégresse générale. En effet, elles apprennent à Marthésie que ses deux sœurs ne sont pas décédées, mais que Marcassin a plutôt été victime d'hallucinations.

74. Nous avons repéré une vingtaine de gravures qui présentent soit une poitrine dénudée de femme (ou un vêtement léger la révélant sans trop faire appel à l'imagination), soit un homme nu... sans que cela soit explicitement précisé dans le texte.

75. A. Audy-Trottier et K. Gladu, art. cité, p. 137. 
Selon A. Defrance, il serait possible de ranger parmi ces apparences trompeuses «la crainte et la répulsion féminines, puisque le marcassin s'est avéré être, contre toute attente, un mari satisfaisant pour celle qui l'a enfin accepté $^{76} »$. Cette interprétation nous permet de proposer une explication pour l'index levé de Marthésie sur le dessin de Marillier. Il se pourrait bien encore une fois que l'illustrateur ait voulu mettre dans la bouche de l'héroïne les mots qu'elle avait prononcés bien avant, peu après son arrivée dans la grotte, la démarcassination de son époux, et ainsi atténuer la tristesse qui précède l'arrivée des fées : «Quel que vous puissiez devenir, mon cher prince, $[\ldots]$ je ne changerai jamais pour vous; il me demeurera toujours une idée charmante de votre métamorphose.» (CDF, IV, p. 372-373) Encore une fois, le dessin de Marillier signifie en renvoyant à la moralité finale du conte, l'érotisation de la scène venant en quelque sorte sublimer la violence et les crimes passés du Prince :

Le plus grand effort de courage,

Lorsque l'on est bien amoureux,

Est de pouvoir cacher à l'objet de ses vœux

Ce qu’à dissimuler le devoir nous engage [...]. ( $C D F, \mathrm{IV}, \mathrm{p}$. 377)

Mais, du même coup, la naturalisation de la métamorphose, qui permet plutôt simultanément d'en "atténuer les résonances sexuelles ${ }^{77}$ ", ne suggèret-elle pas implicitement une certaine égalité homme-femme? En somme, pour rejoindre A. Defrance, l'impression qui se dégage de cette traduction iconographique est compatible avec une conception égalitaire de l'humanimalité que l'on pourrait résumer ainsi : les «hallucinations que nous nommons fantasmes et qui mettent en scène des représentations agressives du rapport sexuel ${ }^{78}$ » existent bel et bien tant chez l'homme que chez la femme, laquelle semble ici s'affirmer, l'index tendu vers son Prince désillusionné.

\section{Signifier et suggérer}

Tout comme le «moment» de la métamorphose laisse des traces le plus souvent implicites — «c'est la réussite de la quête du héros qui suggère que

76. A. Defrance, ouvr. cité, p. I24.

77. A. Gaillard, ouvr. cité, p. 354. L’auteure emploie l'expression «métamorphose naturalisée» dans tous les cas où le personnage n'est pas transformé en animal, mais se déguise en revêtant par exemple une peau. "Le Prince Marcassin" constitue un cas intéressant où ces deux cas de figure se superposent : il s'agit d'une véritable métamorphose en sanglier, mais le retour à la forme humaine se trouve euphémisée par la naturalisation du processus.

78. A. Defrance, ouvr. cité, p. I25. 
les données ont évolué 79 — - les illustrations de Marillier que nous avons analysées ne font pas que reproduire explicitement le texte. À la lumière de ce trop bref survol, il serait présomptueux de proposer une poétique uniforme de l'iconographie animalière des contes de Madame d'Aulnoy, voire de l'ensemble de celle qui se retrouve dans Le Cabinet des fées. Chose certaine, ces illustrations mettent en lumière l'importance du phénomène de la métamorphose animale dans l'économie des contes de Madame d'Aulnoy. Même si la fonction première de l'image devrait être de "donner à voir les "merveilles" (mirabilia) du récit ${ }^{80}$ ", ce n'est pas le côté spectaculaire du processus en action qui intéresse ici l'illustrateur, mais le parcours que le héros a pu accomplir avant de retrouver sa forme humaine (et l'implicite qui se camoufle sous la sobriété apparente de la représentation). Ces gravures semblent donc répondre "au désir du lecteur d'être à la fois satisfait dans ses attentes vis-à-vis d'une œuvre qu'il veut "reconnaître", et surpris par une nouveauté en décalage (mesuré) avec ses attentes ${ }^{8 \mathrm{I}}{ }$. Inscrite au cœur d'un réseau de tensions interprétatives, l'image semble, d'une part, signifier et accomplir une fonction "pédagogique, morale, universelle ${ }^{82}$ ", où «il s'agit de fixer dans l'esprit du lecteur, sous forme imagée, la mémoire d'un énoncé discursif ${ }^{83}$ » qui a trait avec la moralité finale du conte. D'autre part, résultat probable de l'influence des peintres dans le domaine du livre illustré dans la seconde moitié du XVIII ${ }^{\mathrm{e}}$ siècle, se profile dans ces gravures une esthétique où l'image viendrait pour ainsi dire modifier la trajectoire du texte écrit : «Loin de concurrencer le texte, l'image, feignant de se soumettre à lui, le complète, l'amplifie, le déforme, et parfois le corrompt ${ }^{84}$ », ou encore le subvertit, venant suggérer, comme nous l'avons démontré, une interprétation qui convoque l'implicite. Ces représentations liées à la métamorphose animale, qui peuvent sembler de prime abord en marge du cœur de l'action dramatique des contes, procèdent toutefois d'une bipolarité dont la richesse heuristique n'a pas fini d'être épuisée. À la fois lieu d'une représentation de l'archaïque et mise à distance de scènes qui permettent aux illustrations de fonctionner comme tableaux, autorisant cette pause interprétative plus difficile lors de la seule lecture du texte, les dessins de Marillier favorisent la lecture transgressive des scènes, et rejoignent

79. A. Gaillard, ouvr. cité, p. 346 [nous soulignons].

80. C. Martin, «L'illustration du conte de fées (I697-1789)», art. cité, p. II8.

8I. M. Levacher, art. cité, p. 36.

82. A.-M. Bassy, art. cité, p. I49.

83. Ibid., p. I52.

84. Ibid., p. I54. 
ainsi la subversion qui sous-tend les contes de Madame d'Aulnoy, rendant l'interprétation autant "ambiguë» que "perverse» ${ }^{85}$.

Cela dit, ces gravures d'une qualité esthétique remarquable, qui viennent se superposer à des textes écrits un siècle auparavant alors que l'illustration du conte de fées "demeure fruste, maladroite, à cent lieux du merveilleux ${ }^{86}$ ", font nécessairement écho aux images qui ornent les romans illustrés de la fin du XVIII ${ }^{\mathrm{e}}$ siècle, "au point qu'on en retrouve quasiment tous les topö̈ (images galantes, scènes d'effraction, instants théâtralisés de la surprise, de la reconnaissance, postures pathétiques... ${ }^{87}$ ». En effet, le choix des scènes représentées, de même que l'esthétique qui les caractérise, participent du même principe sous-jacent à la traduction opérée par l'éditeur et l'illustrateur, qui semblent avoir voulu davantage susciter l'émotion du lecteur que représenter le merveilleux. Alors que l'iconographie de fin du XVIII ${ }^{\mathrm{e}}$ siècle est investie par la sensibilité, l'utilisation de tableaux pathétiques sert à actualiser une ambition morale: "[celle] d'orienter la lecture d'une manière propre à rendre sensible une conception de la fiction en général, et des contes merveilleux en particulier ${ }^{88}$ ". Notre analyse a montré que la traduction offerte par Marillier semble avoir comme ambition, ainsi que l'a suggéré Nathalie Ferrand, d' "illustrer un texte en l'éclairant d'une lumière différente ${ }^{89}$ ", grâce au langage de l'image, devenu celui de l'émotion et de la sensualité. Il serait éventuellement intéressant de poursuivre l'analyse en se penchant sur un autre conte de Madame d'Aulnoy, illustré par Marillier dans Le Cabinet des fées, et qui relève plutôt d'une représentation positive de l'animalité et de la fiancée-animal. Mentionnons simplement pour l'instant que le dessin qui accompagne La Biche au bois semble tout aussi bien montrer la théâtralisation pathétique d'une scène-clé dans laquelle, cette fois, la femme métamorphosée est devenue l'animal désirable.

Dans l'illustration que propose Marillier, représentant le moment où le roi vient de blesser la biche, les symboles phalliques (épée, flèches) traduisent ici l'agressivité masculine qui - contrairement aux autres contes que nous avons analysés, dans lesquels l'amour du personnage féminin permettait la déserpentination ou la démarcassination de l'homme - est à la source de la métamorphose. Si, malgré l'inversion des rôles, c'est ici la

85. Ibid., p. 154 .

86. Ibid., p. I4I.

87. C. Martin, "L'illustration du conte de fées (I697-I789)", art. cité, p. II5.

88. A. Audy-Trottier et K. Gladu, art. cité, p. I44.

89. N. Ferrand, «Introduction : l'illustration littéraire à l'épreuve de la traduction», dans N. Ferrand (dir.), Traduire et illustrer le roman au XVIII ${ }^{e}$ siècle, 20II, p. I. 
violence qui rend «l'accès à la féminité possible et définitif $9{ }^{\circ}$ ", on ne peut être qu'étonné que toute trace de sang ait été évacuée de la représentation. Revenue à son gîte et ayant repris son apparence humaine à la tombée du jour, Désirée reçoit les soins de sa suivante Giroflée et se fait bander le bras «dont le sang coulait avec abondance» (CDF, III, p. 425).

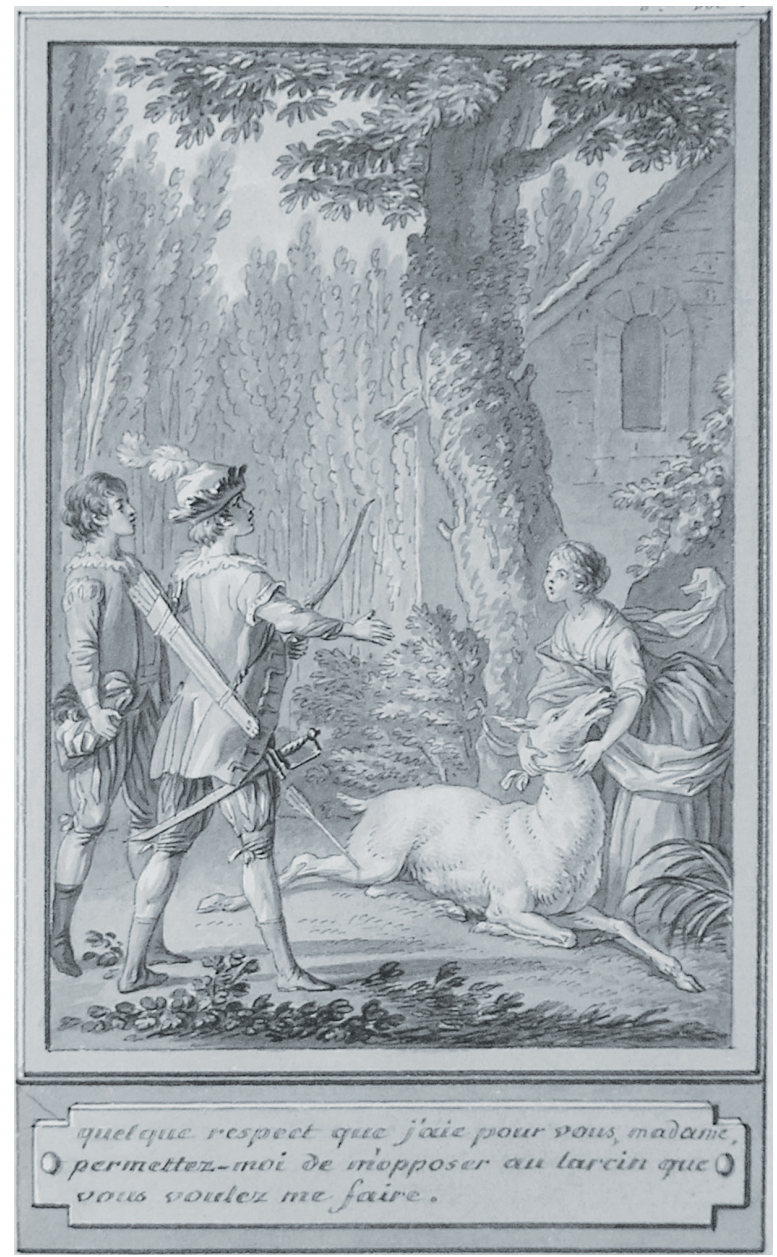

Fig. 3. — «La Biche au bois» tiré du Cabinet des fées chez Manget \& Cie, Genève, 1785 .

Collection privée.

90. A. Defrance, ouvr. cité, p. 139. 
Pourtant Marillier se contente de présenter une flèche bien propre enfoncée dans le grasset droit de l'animal, ce qui semble oblitérer une partie de la symbolique sexuelle - la perte de la virginité. Encore une fois, s'agit-il d'une lecture superficielle, ou d'une "traduction" permettant de marquer le fait que la blessure est perçue dans le texte - ce que permet de soutenir la gueule mi-ouverte et le regard extasié de la biche — comme non dénuée de plaisir? : «[...] elle ne pouvait s'empêcher d'aimer un mal qui lui procurait tant de bien" (CDF, III, p. 428).

Quoi qu'il en soit, la métamorphose animale, si elle permet d'une part à Madame d'Aulnoy de rendre possible la représentation des interdits et de transgresser les bienséances, offre d'autre part à l'éditeur et à l'illustrateur du Cabinet des fées, un siècle et demi après la rédaction du texte, la possibilité d'opérer une traduction oscillant entre le didactique à structure impérative forte que l'on retrouvait jadis dans les emblèmes, et le polysémique dont le sens plus littéraire colorait par exemple les fables. Ces illustrations témoignent également d'un moyen parallèle au texte qui permet aussi de "s'adresser à l'adulte comme à un enfant», et de "pointer l'immaturité de tout homme, sa bestialité»" "11, ce qui rejoint tout à fait la subversion caractéristique de l'écriture de Madame d'Aulnoy. La représentation des monstres est exceptionnelle dans Le Cabinet des fées: hormis Serpentin Vert, la seule autre occurrence est le dragon qui apparaît sur la planche associée à l'«Histoire du Prince engageant» (Cat. 97, CDF 32-I) de Thomas-Simon Gueullette. Cette répugnance à leur représentation, la réduction fréquente de la taille des animaux terrifiants, de même que l'euphémisation de certains tableaux où on s'abstient de montrer la laideur physique ou le sang qui coule, toutes ces caractéristiques participent bel et bien de «l'appel du mièvre ${ }^{92}$ » auquel a répondu Marillier. Selon Stéphane Lojkine, l'illustrateur aurait ainsi joué un rôle essentiel dans cette "véritable conversion de la représentation", alors que «l'ensemble de la culture [...] change d'âge, et va utiliser l'enfance comme supplément des bienséances effondrées " ${ }^{33}$. Certes, même si l'on n'y retrouve pas l'obscénité caractéristique des illustrations de romans libertins (Thérèse philosophe par exemple), il reste que les dessins de Marillier montrent que cet «appel du mièvre» ne s'est pas actualisé de façon linéaire ou instantanée. Au moment où se constitue un lectorat enfantin, il semble difficile d'intégrer pleinement ces représentations érotisées (explicitement ou encore soumises à un

91. A. Gaillard, ouvr. cité, p. 325.

92. S. Lojkine, «Marillier, l'appel du mièvre», dans A. Zygel-Basso (dir.), ouvr. cité, p. 427-449.

93. Ibid., p. 428-429. 
encodage galant), de même que les nombreuses scènes de décollation qui parsèment les planches du Cabinet des fées, à une quelconque ébauche des canons de l'illustration pour la jeunesse. Nous en sommes bel et bien aux balbutiements de cette "conversion de la représentation", en amont d'une évolution qui accompagnera, au début du XIX ${ }^{\mathrm{e}}$ siècle, «l'infantilisation du genre $^{94} »$. De plus, par le va-et-vient obligé entre le texte et l'image, les illustrations nous ont semblé exemplifier, de manière emblématique, les caractéristiques du déroulement temporel du récit fabuleux qui procédent du motif de la métamorphose animale, telles que suggérées par Aurélia Gaillard : temps discontinu et élastique, temps rédimé et temps heuristique ${ }^{95}$. Enfin, si l'on accepte, à la suite de Pascal Ory, l'existence d'un «imaginaire social, définissable comme le lien unissant entre elles un certain nombre de représentations pour leur donner sens", tout en assumant qu'il s'agit là d'imageries qui n'existent qu'à travers le regard a posteriori du chercheur et non de systèmes qui avaient jadis "une cohérence contemporaine ${ }^{96}$, nous nous croyons autorisé, au terme de ce survol, à rejoindre la séduisante conclusion à laquelle était parvenue Catherine Velay-Vallantin à propos des illustrations accompagnant "Barbe-Bleue» dans Le Cabinet des fées : à savoir que l'illustrateur (et le lecteur) finissent parfois par voir "un "château" qui n'existe pas ${ }^{97}$ » dans le texte... ainsi, par exemple le sein découvert de Marthésie, la beauté de Laidronette, ou ailleurs, cette biche transpercée, mais qui oublie de saigner.

\section{Bibliographie}

Audy-Trottier Andréane et Gladu Kim, «Le discours des éditeurs ou le rôle pédagogique de l'imagination: le Cabinet des fées et les Voyages imaginaires", dans A. Zygel-Basso (dir.), Imager la Romancie. Dessins de Clément-Pierre Marillier pour Le Cabinet des fées et Les Voyages imaginaires (I785-I789), Paris, Hermann, "Collections de la République des Lettres (Série Sources)", 2013, p. I25-I44.

Aulnoy Madame d', L'oiseau bleu et autres contes, illustrations de F. Clément, Paris, Grasset, coll. "Grasset-Jeunesse», I99I.

94. O. Piffault, «Éditer la féerie : postérité et concurrents du Cabinet des fées», dans O. Piffault (dir.), ouvr. cité, p. I40.

95. A. Gaillard, ouvr. cité., p. 355.

96. P. Ory, L'histoire culturelle, 2004, p. 96.

97. C. Velay-Vallantin, «Barbe-Bleue, le dit, l'écrit, le représenté», Romantisme, n 78, 1992, p. 8 . 
BAssy Alain-Marie, "Le texte et l'image», dans H.-J. Martin et R. Chartier (dir.), Histoire de l'édition française. Le livre triomphant I660-I830, Paris, Promodis, I984, t. II, p. I4O-I6I.

Bettelheim Bruno, Psychanalyse des contes de fées, traduit de l'américain par T. Carlier, Paris, Robert Laffont, coll. "Livre de Poche / Pluriel», I979 [1976].

Bloch Jeanne, «Le héros animal dans les contes de fées de $\mathrm{M}^{\mathrm{me}} \mathrm{d}^{\text {'Aulnoy. }}$ Le Prince Marcassin, Serpentin Vert, La Chatte blanche, La Biche au bois", Dix-huitième siècle, $\mathrm{n}^{\circ}$ 42, 2010, p. II9-138.

Cheilan Liliane, "La Bête et le Prince dans quelques versions illustrées ou versions en images de La Belle et la Bête», dans J. Chiron et C. Seth (dir.), Marie Leprince de Beaumont. De l'éducation des filles à La Belle et la Bête, Paris, Classiques Garnier, 2013, p. I75-188.

Chevalier Jean et Gheerbrant Alain, «Dragon", Dictionnaire des symboles, Paris, Robert Laffont / Jupiter, coll. «Bouquins», I982 [1969], p. 366-369.

Daubenton Louis-Jean-Marie, "Dragon, draco, (Hist. natur. Zoolog.)», dans Encyclopédie ou Dictionnaire raisonné des sciences, arts et des métiers, Stuttgart-Bad Cannstatt, Friedrich Frommann Verlag (Günther Holzboog), I966 [réimpression de l'édition de Paris-Neuchâtel de I75II780], t. V [I755], p. 504.

Defrance Anne, Les contes de fées et les nouvelles de Madame d'Aulnoy (I690-I698). L'imaginaire féminin à rebours de la tradition, Genève, Droz, 1998.

FerRand Nathalie, Livres vus, livres lus : une traversée du roman illustré des Lumières, Oxford, Voltaire Foundation, coll. "SVEC», 2009, nº 3.

- (dir.), Traduire et illustrer le roman au XVIII siècle, Oxford, Voltaire Foundation, coll. «SVEC», 20II, $\mathrm{n}^{\circ} 5$.

Gaillard Aurélia, Fables, mythes, contes. L'esthétique de la fable et du fabuleux (I660-I724), Paris, H. Champion, coll. «Lumière Classique», 1996.

Guillerm Alain, "Le système de l'iconographie galante», Dix-huitième siècle, $\mathrm{n}^{\circ} \mathrm{I} 2$, $\mathrm{1} 98 \mathrm{O}$, p. I77-194.

Ionescu Christina (dir.), Book Illustration in the Long Eighteenth Century. Reconfiguring the Visual Periphery of the Text, Newcastle upon Tyne, Cambridge Scholars Publishing, 20II.

Levacher Maëlle, «Les lieux communs dans l'Histoire naturelle de Buffon : rhétorique judiciaire, rivalité dans la récriture et compromis épistémologique", Dix-huitième siècle, ${ }^{\circ}$ 42, 2010, p. 35-54.

-, Buffon et ses lecteurs, Paris, Classiques Garnier, coll. «L'Europe des Lumières", 20II. 
Lewis Ann, Sensibility, reading and illustration. Spectacles and signs in Graffigny, Marivaux and Rousseau, Londres, Legenda, 2009.

LoJkine Stéphane, "Marillier, l'appel du mièvre», dans A. ZygelBasso (dir.), Imager la Romancie. Dessins de Clément-Pierre Marillier pour Le Cabinet des fées et Les Voyages imaginaires (I785-I789), Paris, Hermann, coll. "Collections de la République des Lettres (Série Sources)", 2013, p. 427-449.

Mainil Jean, Madame d'Aulnoy et le rire des fées : essai sur la subversion féerique et le merveilleux comique sous l'Ancien Régime, Paris, Kimé, 200I.

Martin Christophe, "Dangereux Suppléments". Lillustration du roman en France au XVIII siècle, Louvain, Peeters, coll. "La République des Lettres", 2005.

—, "Lillustration du conte de fées (I697-1789)", CAIEF, $\mathrm{n}^{\circ}$ 57, 2005, p. II3-I32.

—, "L'émergence d'un nouvel objet de recherches : le roman illustré au XVIII ${ }^{\mathrm{e}}$ siècle", dans Ph. Stewart et M. Delon (dir.), Le second triomphe du roman du XVIII ${ }^{e}$ siècle, Oxford, Voltaire Foundation, coll. "SVEC», 2009, $\mathrm{n}^{\circ}$ 2, p. 193-204.

Mayer Charles-Joseph de (éd.), Le Cabinet des fées ou Collection des contes defées, et autres contes merveilleux ornés de figures, Genève, Barde, Manget \& Cie, à Paris chez Cuchet, vol. III et IV, I785.

Meunier Véronique, "Le bestiaire fantastique», dans O. Piffault (dir.), Il était une fois... les contes de fées, Paris, Seuil / BNF, 200I, p. 374-377.

Ory Pascal, L'histoire culturelle, Paris, PUF, 2004.

Paradis Swann, "Signifier ou suggérer? Sobriété d'une faune fabuleuse», dans A. Zygel-Basso (dir.), Imager la Romancie. Dessins de ClémentPierre Marillier pour Le Cabinet des fées et Les Voyages imaginaires (I785-I789), Paris, Hermann, coll. "Collections de la République des Lettres - Série Sources", 20I3, p. 345-373.

Picaud Carine, "Lillustration du conte de fées», dans O. Piffault (dir.), Il était une fois... les contes de fées, Paris, Seuil /BNF, 200I, p. I58-I62.

Picaud Carine et Piffault Olivier, Contes de fées en images. Entre peur et enchantement, Paris, Éditions de La Martinière, 20I3.

Piffault Olivier, "Éditer la féerie : postérité et concurrents du Cabinet des fées", dans O. Piffault (dir.), Il était une fois... les contes de fées, Paris, Seuil/BNF, 200I, p. I35-I53.

Pinon Laurent, Livres de zoologie de la Renaissance: une anthologie (I450I700), Paris, Klincksieck, coll. «Corpus iconographique de l'histoire du livre», 1995 . 
Роттs Alex, «Natural order and the call of the wild: the politics of animal picturing", The Oxford Journal, vol. I3, n ${ }^{\circ}$ I, 1990, p. I2-32.

Rousset Jean, Passages, échanges et transpositions, Paris, José Corti, I990.

Slater Maya, "Les animaux parlants dans les Contes de fées de $\mathrm{M}^{\mathrm{me}}$ d'Aulnoy", dans J. Perrot (dir.), Tricentenaire Charles Perrault. Les grands contes du XVII siècle et leur fortune littéraire, Paris, In Press, coll. "Lectures d'enfance», I998, p. I57-I64.

Stewart Philip, Engraven Desire, Durham-Londres, Duke University Press, I992.

—, "Images de ce qui ne fut jamais", dans R. Jomand-Baudry et J.-F. Perrin (dir.), Le conte merveilleux au XVIII siècle. Une poétique expérimentale, Paris, Kimé, 2002, p. 370-399.

Thirard Marie-Agnès, Les contes de fées de Madame d'Aulnoy, une écriture de la subversion, thèse de doctorat, Université Lille 3, I994.

Velay-Vallantin Catherine, «Barbe-Bleue, le dit, l'écrit, le représenté», Romantisme, $\mathrm{n}^{\circ}$ 78, 1992, p. 75-90.

Warner Marina, Fantastic Metamorphoses, Other Worlds. Ways of Telling the Self, Oxford, Oxford University Press, 2002.

Zygel-Basso Aurélie (dir.), Imager la Romancie. Dessins de Clément-Pierre Marillier pour Le Cabinet des fées et Les Voyages imaginaires (I785I789), Paris, Hermann, coll. "de la République des Lettres - Série Sources)", 2013. 